\title{
Intracerebral Hemorrhage
}

\author{
Siniša Franjić* \\ Independent Researcher, Croatia \\ *Corresponding Author: Siniša Franjić, Independent Researcher, Croatia.
}

\begin{abstract}
Intracerebral hemorrhage begins suddenly with a headache followed by signs of a steady increase in neurological losses such as weakness, inability to move, insensitivity, loss of speech or vision, and confusion. Nausea, vomiting, epileptic seizures and loss of consciousness are common and may occur within a few minutes. Physicians often make a diagnosis of intracerebral hemorrhage without requiring any examination, while computed tomography $(C T)$ or magnetic resonance imaging (MRI) are generally have done when physicians suspect a stroke. Both imaging can help physicians differentiate ischemic stroke from stroke-related stroke. Scans can also indicate the extent of brain tissue damage and whether there is elevated pressure in other areas of the brain.
\end{abstract}

Keywords: Brain, Hemorrhage, Stroke, CT, MRI

\section{INTRODUCTION}

The aim of a neurological history and examination is to locate the area of damage within the nervous system and then, on the basis of the tempo of the history, to make an attempt at diagnosis [1].

The nervous system comprises the cerebral cortex, brain stem, cerebellum, spinal cord, roots and peripheral nerves. The term 'neuraxis' is often used to encompass the cerebral cortex, brain stem, cerebellum and spinal cord, alternatively known as the central nervous system (CNS) in contradistinction to the spinal nerve roots and the nerves which they eventually form, the neuromuscular junction and muscle, which collectively is known as the peripheral nervous system (PNS).

Information is received into the brain via the senses (vision, hearing, smell, touch). The response from the nervous system to stimuli perceived by the senses is either in the form of speech or movement. On this basis, there are therefore major ascending pathways within the spinal cord, brain stem and cortex which convey information from the periphery to the brain.

Similarly, there are major pathways within the brain conveying information from vision to the appropriate part of the brain which deals with the vision in the occipital cortex. The major motor pathway runs from the cortex down through the brain stem and spinal cord, and ends on the anterior horn cells on the peripheral nerves. Activation of this pathway produces movement.

The brain can therefore be considered as a 'wiring diagram' with descending pathways, ascending pathways and horizontal pathways which, within the brain, are the cranial nerves and, in the spinal cord, are the nerve roots. Attempting to make a diagnosis, therefore, is often a matter of identifying the point of intersection between ascending and descending pathways and horizontal pathways.

\section{NEUROLOGICAL SYMPTOMS}

The medical profession in its training as well as in everyday practice takes a fundamentally therapeutic attitude to its patients [2]. The aim is perceived to be the diagnosis and assessment of injury or illness; and its restitution to the maximum extent possible. However, in the medico-legal context the role of the medical expert is quite different. $\mathrm{He}$ is seeing the "patient" in order to evaluate the effect that injury has caused and to provide a prognosis which will allow the court to come to a decision about the magnitude of compensation. By the time most claims come to settlement therapeutic aspects are generally long past. The practical therapeutic approach of medicine therefore may lead to conflicts which should be perceived by the doctor.

In the assessment of any patient complaining of neurological symptoms some general principles must be borne in mind. Neurological symptoms 
may be associated with objective abnormality demonstrable on examination. But the objective signs may be disproportionate, that is, they may be less than the symptoms would lead you to expect so that wilful or subconscious exaggeration is suspected. Or the signs may seem unrelated to the symptom, for example, a complaint of paralysis when the signs indicate sensory loss. Or finally the neurological symptoms may have no corresponding sign or abnormality on examination. A lack of objective support for the organic basis of a complaint is relatively common in neurology. It occurs, for example, in many pain syndromes, especially those where the pain is associated with damage to peripheral nerve, the spinal cord or central nervous system. Migraine, epilepsy, irritability and personality change are all common posttraumatic phenomena where there is usually no associated objective abnormality. Compensation gives a motive to perpetuate symptoms previously experienced or to claim symptoms that have either recovered or never been sufficiently severe to cause disability.

The doctor must be able to recognise patterns of symptomatology associated with organic disorder of the CNS and to be able to distinguish these from symptoms that are exaggerated or feigned. Where there are abnormalities demonstrable on examination the case may appear straightforward and convincing but there should be an "appropriateness" between history, present symptoms and objective abnormality.

Despite the large numbers of individuals affected, members of the generalpublic often have little insight into the consequences of a neurological condition until they have direct personal experience [3]. Levels of awareness are low even about relatively common conditions such as epilepsy and head injury. Conditions that are less common, such as Friedreich's ataxia, are largely unheard of and poorly understood by both the general public and health and social care professionals alike. Research commissioned to underpin the National Service Framework (NSF) for Long-term Conditions demonstrates the fear felt by patients who perceive that the professionals organising and delivering their care have little understanding of their particular needs. Individuals with conditions affecting their ability to communicate are particularly vulnerable and often disadvantaged by the failure of staff to recognise the extent to which this problem affects care. Similarly, busy staff may wrongly interpret cognitive and behavioural problems and attribute any difficulties to personality defects, failure to adjust or obstinacy. These labels once acquired are often difficult to shake off, becoming accepted and perpetuated by other health professionals without further investigation or assessment. In this way the ignorance and attitudes of others can compound the difficulties people with neurological conditions experience, often causing them to feel stigmatised and misunderstood, which can, in turn, lead to further social, emotional and economic disadvantage.

Many neurosurgical disease states such as intracerebral hemorrhage, ischemic neurological injury, and carotid artery disease are affected by the blood pressure [4]. Finding an optimal level of blood pressure depends on many factors including severity of chronic hypertension, patient age, and impairment of intracranial compliance (e.g., patients with elevated ICP). Blood pressure control may be particularly critical in patients with (or at risk for) hemorrhage in areas that place neurologic tissue at risk for further injury (e.g., brain or spinal cord).

For individual patients with neurologic injury, the optimal blood pressure range is often difficult to establish. Ideally, the blood pressure range would allow for optimal cerebral perfusion without placing the patient at risk for cerebral hemorrhage or edema. This can be difficult to demonstrate since CT and MRI - the usual scanning methods - are static exams and are impractical for providing information over time. More advanced studies such as PET scanning can give more insight but are not readily available. One study in stroke patients shows a U-shaped blood pressure curve with increased mortality for patients presenting with blood pressures greater than $220 / 111$ or less than $100 / 61$. Hypotension is rare in patients in the acute setting of stroke. When hypotension is observed in this population, potential etiologies include aortic dissection, dehydration, blood loss, sepsis, and decreased cardiac output. If hypotension is noted, immediate steps should be taken to correct the hypotension and to identify and treat the underlying cause.

\section{ITRACRANIAL HEMORRHAGE}

Intracranial hemorrhage ( $\mathrm{ICH})$ is the most feared complication of revascularization with EVT for it is characterized by high rates of morbidity and mortality [5]. The term encompasses several clinical entities that include subarachnoid hemorrhage (SAH) and intraparenchymal hemorrhage (IPH). The presence of ICH following EVT (endovascular 
thrombectomy) is typically categorized clinically as either symptomatic or asymptomatic. Symptomatic ICH (sICH) is defined as the presence of blood anywhere on CT imaging in the setting of clinical deterioration or a four-point drop on the National Institutes of Health Stroke Scale (NIHSS).

In the recent RCTs, sICH was the only consistently reported complication and rates ranged between 0 and $7.7 \%$ for those treated with EVT plus tPA (tissue plasminogen activator) and from 1.9 to $6.4 \%$ for those treated with tPA alone. Meta-analysis of the pooled data from the initial and recent RCTs (randomized controlled trials) found no statistical difference between the two treatment groups, indicating that EVT does not confer an increased risk of sICH when compared with tPA alone. Asymptomatic ICH, on the other hand, is typically discovered incidentally on routine imaging after EVT and is not associated with the development of neurologic exam changes or declines in overall neurologic status. One recent RCT reported the rate of asymptomatic ICH at $16.5 \%$ in the EVT plus tPA group and $10.7 \%$ in the tPA-only group.

Intraparenchymal hemorrhage (IPH), also called intracerebral hemorrhage, is a hemorrhage into the brain tissue itself. In cases of AIS (acute ischemic stroke), IPH tends to occur in the location of the affected vascular territory. Intraoperative injury to the vessel wall leading to arterial perforation during the EVT procedure can result in IPH. Injury of this type can occur as a result of excessive traction on the artery of interest, displacement of the normal vasculature during thrombus removal, or multiple passes to remove tenacious thrombus. The thrombectomy devices themselves, stent retrievers more than aspiration catheters, have a recognized association with intraoperative vessel injury. IPH is also a known complication of reperfusion to ischemic brain, which is unrelated to the EVT procedure itself. Meta-analysis found no significant difference in IPH between EVT and tPA treatment groups suggesting that EVT does not increase the risk in appropriately selected patients. The recent RCTs report rates of IPH that range from 5.8 to $11 \%$ for treatment with EVT plus tPA and 5\% to $9 \%$ for treatment with tPA alone.

\section{ITRACEREBRAL HEMORRHAGE}

The World Health Organization estimates for stroke incidences vary from 50 to 500 per 100,000 depending on region [6]. The vast majority of strokes are ischemic strokes, which result from a clot in the artery that supplies the brain with oxygen-rich blood. A hemorrhagic stroke occurs when an artery in the brain leaks blood or ruptures, placing pressure on brain cells and resulting in brain damage. High blood pressure and aneurysms are common causes of hemorrhagic strokes. Hemorrhagic strokes can be intracerebral, resulting when an artery within the brain bursts and the surrounding tissue is flooded with blood, or subarachnoid, which occurs when the bleeding occurs between the brain and the arachnoid mater.

The most common locations for ICH are: the lobes, basal ganglia, thalamus, pons (and to a lesser extent the other parts of the brainstem, the midbrain and the medulla), and cerebellum [7]. These locations correspond to small perforating arteries branch from large cerebral arteries. It is the bifurcation point of the perforating arteries branching off from the larger arteries that seems most vulnerable to damage. Thus, it is also the most likely point at which some kind of rupture or tear, and consequently bleeding, occurs. It has been observed that there is degeneration of the media and smooth muscles of the arterioles at these points. In general, ICH located in basal ganglia, thalamus, cerebellum and pons is most likely related to HTN, whereas lobar presentations are usually associated with other etiologies and require further work up.

Intracerebral hemorrhage (ICH) is spontaneous nontraumatic bleeding into the brain parenchyma [8]. Annually, approximately 65,000 people in the United States suffer an ICH, which accounts for $10-30 \%$ of all stroke cases across different ethnic groups. ICH is the most fatal and least treatable form of stroke, causing, in addition, severe disability among survivors. Patients with ICH uniformly require ICU management and patients cared for in specialized neurologic intensive care units are less likely to die. Although, as compared to ischemic stroke and subarachnoid hemorrhage, the pace of advances in management of ICH has been slow, recent results of clinical trials of recombinant factor VIIa (fVIIa) in acute ICH have generated excitement.

Based on the underlying pathology of ruptured vessel that originates the bleeding, ICH is classified as primary or secondary. The majority of primary ICH result from a ruptured vessel as a consequence of chronic injury to the small cerebral vessels by sustained hypertension (hypertensive vasculopathy) or abnormal protein deposition (cerebral amyloid angiopathy). 
Secondary causes of ICH include underlying vascular malformations, ruptured saccular aneurysms, coagulation disorders, use of anticoagulants and thrombolytic agents, hemorrhage into a preexisting infarct, brain tumor, or infectious focus, and drug abuse.

A primary intracerebral hemorrhage occurs when a blood vessel bursts causing bleeding into the brain; a subarachnoid haemorrhage is bleeding into the subarachnoid space [9]. Primary intracerebral hemorrhages account for $13 \%$ of all strokes, while $6 \%$ are due to subarachnoid haemorrhages. Of the remaining strokes, $12 \%$ are classified as uncertain in origin.

Initial medical management of the suspected stroke patient is primarily aimed at distinguishing whether the symptoms are due to a vascular event or another cause of neurological disorder with rapid onset. Information on which to base a diagnosis is gathered through comprehensive neurological and systemic assessment and brain imaging.

Ischaemic stroke is responsible for about $80 \%$ of all strokes, intracerebral haemorrhage for $15 \%$, and subarachnoid haemorrhage for 5\% [10]. A transient ischaemic attack (or TIA), has the same symptom complex as a stroke, but with a resolution of these symptoms within $24 \mathrm{~h}$. Most TIAs resolve within $1 \mathrm{~h}$. It is increasingly understood that TIAs and minor strokes represent a continuum of disease; some now suggest that the time-based definition of TIA yield to a tissuebased definition, as approximately one third of people with clinically diagnosed TIAs will actually have structural changes visible on neuroimaging, such as diffusion weighted MRI scanning (DWI). Other terms used in the past to describe stroke and TIA, such as cerebrovascular accident (CVA) and reversible ischaemic neurological deficit (RIND), lack specificity, and have generally fallen out of common use.

Risk factors [11]:

- Age

- History of prior stroke

- Smoking

- Hypertension

- Anticoagulation (e.g., warfarin therapy)

- Cerebral amyloidosis

- Cocaine use

CT

Computed tomography (CT) is widely accepted as the gold standard for imaging intracerebral hemorrhage; however, it has not been rigorously examined in prospective studies, and thus the precise sensitivity and specificity is unknown (limited evidence) [12]. For the evaluation of thrombolytic candidates (exclusion of intracerebral hemorrhage), however, CT is clearly the modality of choice based on strong evidence (level I) from randomized controlled trials. By many measures MR is at least as sensitive as CT in the detection of intracerebral hemorrhage, and it is suspected to be more sensitive during the subacute and chronic phases. A recent study indicates that the sensitivity and accuracy of MR in detecting intraparenchymal hemorrhage is equivalent to $\mathrm{CT}$ even in the hyperacute setting (within $6 \mathrm{~h}$ of ictus) (strong evidence).

It is essential that an imaging study reliably distinguish intracerebral hemorrhage from ischemic stroke because of the divergent management of these two conditions. This is especially critical for patients who present within $3 \mathrm{~h}$ of symptom onset under consideration for thrombolytic therapy. Noncontrast CT is currently the modality of choice for detection of acute intracerebral hemorrhage. Acute hemorrhage appears hyperdense for several days due to the high protein concentration of hemoglobin and retraction of clot, but becomes progressively isodense and then hypodense over a period of weeks to months from breakdown and clearing of the hematoma by macrophages. Rarely acute hemorrhage can be isodense in severely anemic patients with a hematocrit of less than $20 \%$ or $10 \mathrm{~g} / \mathrm{dL}$. Although it has been well accepted that CT can identify intraparenchymal hemorrhage with very high sensitivity, surprisingly few studies have been conducted to support this.

\section{RECANALIZATION}

Cerebral infarction is the result of severe enough ischemia for a sui cient time to result in cell death. The progression toward infarction includes protein synthesis failure, anaerobic metabolism, release of neurotransmitters, energy failure, and ultimately, when the threshold of $<0.15 \mathrm{cc} / \mathrm{gm} / \mathrm{min}$ of blood flow is reached, anoxic depolarization [13]. If hypoperfusion can be remedied quickly, penumbral tissues which were not yet critically hypoperfused can be saved. This is the physiological basis of recanalization therapy with lytics and mechanical agents. Ultrasound and physiological studies have shown that recanalization with intravenous recombinant 
tissue plasminogen activator (IVrt-PA) is associated with tissue salvage and better clinical outcomes. Clinical trials have shown that IV rtPA is associated with better outcomes compared with control groups. Similarly, successful endovascular clot removal, retrieval, and lysis have been associated with better clinical outcomes than persistent arterial occlusion. However, no mechanical system has yet been tested in clinical efficacy studies.

Recanalization, the re-establishment of arterial patency can be assessed by angiography and indirectly by transcranial Doppler ultrasound, and graded. Results may be confusing due to inconsistencies in the application of recanalization rating scales, and therefore, it has been recommended that all trials reporting angiographic outcomes include information on target vessel patency, distal i lling, and capillary phase perfusion. Recanalization relates but is not identical to antegrade reperfusion, which is volume of flow through the previously occluded vessel, and collateral perfusion which represents the volume of flow through collaterals to the ischemic region. Even when recanalization is successful, the region may remain ischemic due to distal emboli. Furthermore, flow may be established too late for some or all of the ischemic tissue to be preserved. Therefore, recanalization can be an important marker of treatment effects, but alone is not sufficient to determine whether a treatment is going to be effective. Subjects with M1 or M2 occlusions had prourokinase or placebo infused directly into the proximal portion of the thrombus, initiated within 6 hours of stroke symptom onset. Both groups received heparin. The primary efficacy outcome was recanalization of the M1 or M2 2 hours at er the initiation of the treatment. There was also a primary safety outcome: symptomatic intracerebral hemorrhage within 24 hour of treatment.

\section{MANAGEMENT}

ICH is a medical emergency and mandates prompt diagnosis as any delay in treatment will worsen the outcome [14]. The risk of cardiopulmonary instability and rapid neurological deterioration in the first hours after symptom onset is high. Airway support, blood pressure (BP) control, intracranial pressure (ICP) treatment, reversal of anticoagulation and neurosurgical consultation are often initiated in the emergency department. As in any acutely unstable patient, the initial attention should be directed at the 'ABCs' of emergency therapy.
About one third of patients with supratentorial hemorrhage and the majority with brainstem or cerebellar hemorrhage have either decreased consciousness or bulbar muscle dysfunction requiring early endotracheal intubation. Generally, intubation is indicated for patients with a Glasgow Coma Scale (GCS) score $£ 8$ or in those with rapidly worsening level of consciousness. This is best achieved with shortacting agents such as propofol or etomidate that will prevent transient increases in ICP caused by tracheal stimulation, without prolonged effects on the level of consciousness or neurological function. If circulatory resuscitation is required, isotonic fluids should be given. Hypotonic fluids can exacerbate cerebral edema and increase ICP because free water accumulates in the injured brain tissue. Dextrose-containing solutions should be avoided as hyperglycemia is detrimental to ICH patients (IV/C).

Patients with primary intracerebral haemorrhage presenting within the first $6 \mathrm{~h}$ of the onset and with a systolic blood pressure of over 150 $\mathrm{mmHg}$ should have their blood pressure lowered to $140 \mathrm{mmHg}$ for at least 7 days unless there is any contraindication [15]. Patients with ischaemic stroke should have their blood pressure kept below 185/110 $\mathrm{mmHg}$. Screen forswallowing abnormalities before any food or fluid is given and certainly within $4 \mathrm{~h}$ of admission. This should be done using a standardized screening protocol such as one that first checks the ability of the patient to cough and then goes on to test the ability to swallow teaspoons of water, followed by a glass of water. If a patient is unable to swallow safely, start feeding with a nasogastric tube within $24 \mathrm{~h}$ of admission. If intravenous fluids are required (and enteral hydration is preferred) then avoid the use of glucose solutions as hyperglycaemia may worsen outcomes. Venous thromboembolism is common. Not use anticoagulants even in low dose for prophylaxis. Evidence shows that intermittent pneumatic compression devices are safe and effective at preventing DVT in patients with hemiparetic legs. TED stockings (both short and long) should not be used. At best they are useless, uncomfortable and expensive, at worst harmful. Patients should be carefully monitored for infection, which should be treated early. This requires monitoring of temperature, pulse, blood pressure and oxygen saturation, atleast daily examination ofthe chest, and monitoring for urinary tract infection. There is no evidence to support the use of prophylactic antibiotics after stroke. 
Depression is very common after stroke and can be difficult to identify, particularly in dysphasic patients. All patients should be screened for depression. Cognitive behavioural therapy is usually the treatment of first choice.

\section{CONCLUSION}

Intracerebral hemorrhage is a type of stroke caused by bleeding within the brain tissue itself. This condition is extremely dangerous for human life. A stroke occurs when the brain is deprived of oxygen due to a break in blood flow. Intracerebral hemorrhage is most commonly caused by hypertension, arteriovenous malformations, or head injuries. Treatment mainly focuses on stopping bleeding, removing a blood clot and reduction pressure on the brain.

\section{REFERENCES}

[1] Cumming, W. J. K. (1998.): „, Neurology“, Cavendish Publishing Limited, London, UK, pp. 1. -3 .

[2] Godwin-Austen, R. B. (1991.): „, Neurology“ in Jackson, J. P. (ed): „A Practical Guide to Medicine and the Law“, Springer-Verlag, London, UK, pp. 137. - 138.

[3] Peniket, D.; Grove, R. (2007.): „, Rehabilitation of Patients with an Acquired Brain Injury or a Degenerative Neuromuscular Disorder" in Jester, R. (ed): „Advancing Practice in Rehabilitation Nursing", Blackwell Publishing, Oxford, UK, pp. 125. - 126.

[4] Concannon, D. M. (2019.): „, Neurocriminology - Forensic and Legal Applications, Public Policy Implications“, CRC Press, Taylor \& Francis Group, Boca Raton, USA, pp. 46.

[5] Lund, P. E.; Kirsch, J. R. (2012.): ”, Cardiovascular Risk and Instability: Evaluation, Management, and Triage" in Brambrink, A. M.; Kirsch, J. R. (eds): „Essentials of Neurosurgical Anesthesia \& Critical Care - Strategies for Prevention, Early Detection, and Successful Management of Perioperative Complications", Springer Science+Business Media, LLC, New York, USA, pp. 73. -74.

[6] Chartrain, A. G.; Awad, A. J.; Mocco, J. (2018.): „ Principles for Complication Avoidance and Management in Thrombectomy for Ischemic Stroke" in Gandhi, C. D.; Prestigiacomo, C. J. (eds): „Cerebrovascular and Endovascular Neurosurgery - Complication Avoidance and Management", Springer International Publishing AG, Cham, Switzerland, pp. 382. -383.

[7] Feen, E. S.; Lavery, A. W.; Suarez, J. I. (2004.): „ Management of Nontraumatic Intracerebral Hemorrhage" in Suarez, J. I. (ed): "Critical Care Neurology and Neurosurgery“, Humana Press Inc., Totowa, USA, pp. 355.

[8] Rost, N.; Rosand, J. (2010.): „Intracerebral Hemorrhage" in Torbey, M. T. (ed): , Neurocritical Care", Cambridge University Press, Cambridge, UK, pp. 143.

[9] Chambers, T. (2007.): „Stroke Care“ in Jester, R. (ed): „Advancing Practice in Rehabilitation Nursing", Blackwell Publishing Ltd, Oxford, UK, pp. 109.

[10] Gubitz, G. (2007.): „Acute stroke management and prevention of recurrences" in Candelise, L.; Hughes, R.; Liberati, A.; Uitdehaag, B. M. J.; Warlow, C. (eds): „Evidence-Based Neurology - Management of Neurological Disorders", BMJ Books, Blackwell Publishing, Malden, USA, pp. 113.

[11] Stead, L. G.; Stead, S. M.; Kaufman, M. S. (2006.): „FIRST AID Emergency Medicine Clerkship, Second Edition“, The McGraw-Hill Companies, Inc., New York, USA, pp. 90.

[12] Vo, K. D.; Lin, W.; Lee, J. M. (2011.): „, Neuroimaging in Acute Ischemic Stroke" in Medina, L. S.; Blackmore, C. C.; Applegate, K. E. (eds): „Evidence-Based Imaging - Improving the Quality of Imaging in Patient Care", Springer Science+Business Media, LLC, New York, USA, pp. 185.

[13] Brown, D. L.; Johnston, K. C.; Palesch, Y. Y. (2012.): „Acute ischemic stroke“ in Ravina, B.; Cummings, J.; McDermot, M. P.; Poole, R. M. (eds): „Clinical Trials in Neurology - Design, Conduct, Analysis", Cambridge University Press, Cambridge, UK, pp. 242. - 243.

[14] Voetsch, B.; Kase, C. S. (2011.): „Management of spontaneous intracerebral hemorrhage" in Biller, J.; Ferro, J. M. (eds): „Evidence-Based Management of Stroke“, tfm Publishing Limited, Shrewsburry, UK, pp. 176.

[15] Rudd, T.; Bhalla, A. (2018.): „Stroke“ in Sprigings, D.; Chambers, J. G. (eds): „Acute Medicine - A Practical Guide to the Management of Medical Emergencies, Fifth Edition", John Wiley \& Sons Ltd, Chichester, UK, pp. 408 .

Citation: Siniša Franjić, "Intracerebral Hemorrhage", International Journal of Research Studies in Medical and Health Sciences. 2020; 5(11): 25-30.

Copyright: (0) 2020 Siniša Franjić, This is an open-access article distributed under the terms of the Creative Commons Attribution License, which permits unrestricted use, distribution, and reproduction in any medium, provided the original author and source are credited. 